\title{
On Formulation and Training of Grey-box Thermal Model for Low-rise Residential Buildings
}

\author{
Zixiao Shi ${ }^{1}$, Guy Newsham ${ }^{1}$, Ajit Pardasani ${ }^{1}$, H. Burak Gunay ${ }^{2}$ \\ ${ }^{1}$ National Research Council Canada, Ottawa, Ontario, Canada \\ ${ }^{2}$ Carleton University, Ottawa, Ontario, Canada
}

\begin{abstract}
This paper details the methodologies for creating and training grey-box thermal models for low-rise residential buildings. This paper covers different aspects of model development such as model vectorization, cost function definition and parameter estimation. Different computing strategies such as GPU accelerated calculation are also explored. This paper also briefly demonstrates the possibility of rearranging the model equation for predictive control purposes. The performance of an example grey-box model is tested against common datadriven machine learning models using a dataset of over 500 dwellings. Overall the grey-box model achieved good temperature prediction accuracy at a 4-hour forecast horizon and produced meaningful insights from the estimated parameters. The possibilities of using grey-box models for more advanced applications, such as model predictive control and remote auditing are also discussed.
\end{abstract}

\section{Introduction}

Recent advancement in smart home and smart grid infrastructure has led to a significant growth in the data collection capabilities in low-rise residential buildings. This leads to the possibility of training tailored thermal models using data driven methods for each individual household. These models can be used in a wide variety of applications, such as model predictive controls for heating/cooling, fault detection, and dwelling characteristics extraction.

Three categories of models can be adopted to quantify building thermal processes: first-principle models, greybox models and black-box models. First principle models are derived from fundamental physical phenomena and are usually used for detailed forward simulation with predefined model parameters. While some authors have tried using first principle building simulation engines such as EnergyPlus and esp-r to automatically generate models of existing buildings (Eisenhower, O’Neill, Narayanan, Fonoberov, \& Mezić, 2012), this task is still quite challenging due to the high number of parameters that need to be defined/estimated inside the first-principle models.

Grey-box models are simplified models loosely based on first principles. They have a reduced number of parameters and equations, and are flexible to different engineering applications (Déqué, Ollivier, \& Poblador, 2000). Grey-box models can be obtained through a model reduction process from first-principle models, or created manually from expert knowledge.

The adoption of quantitative grey-box thermal models for commercial buildings has been widely researched, yet its application on individual residential dwellings is still rare (Déqué et al., 2000; Gianniou, Reinhart, Hsu, Heller, \& Rode, 2018). Originally grey-box models were used as surrogate models to detailed building performance simulation (Crawley, Sander, Cornick, \& Newsham, 1993). Later researchers found it possible to train greybox models using data-driven approaches to represent individual buildings. Some of these applications include an R-C model used by Braun and Chaturvedi (2002), and state-space representation using physical parameters (Molina, Lu, Sherman, \& Harley, 2013).

A black-box model, as its name suggests, is usually applied without the need to explicitly define its model structure or parameters except for a limit number of hyper parameters. In recent years, many researchers have started to use black-box models derived from statistics and machine learning to predict and monitor building thermal response. Examples of these applications include artificial neural networks (Mustafaraj, Lowry, \& Chen, 2011) and support vector machines (Li, Meng, Cai, Yoshino, \& Mochida, 2009). While black-box models may provide solutions for complex building thermal processes where grey-box models fail, one major disadvantage is their inability to extrapolate beyond their training target. Further, unlike grey-box models, the parameters derived from trained black-box models retain little physical meaning, and black-box models cannot be rearranged to perform other tasks (Shi \& O’Brien, 2019).

\section{Research objective}

The goal of this research is to investigate methods to formulate grey-box thermal models for low-rise residential dwellings. Such models can be used for scalable model predictive control, demand response and remote auditing applications. The model template for this research is based on previous grey-box modelling efforts (Braun \& Chaturvedi, 2002; Gunay, Bursill, Huchuk, O'Brien, \& Beausoleil-Morrison, 2014; Wang \& Xu, 2006). The proposed grey-box model is capable of providing reliable short-term (1 to 4 hours) thermal response forecasts as well as estimating reduced thermal parameters. Ways of compiling and computing these models are also explored. Performance of the model is compared against machine learning models using a dataset of over 500 residential dwellings. 


\section{Methodology}

This section discusses the proposed grey-box modelling scheme. Formulation of the grey-box model and comparison with other modelling alternatives is discussed first, followed by an explanation of the feature selection processes and vectorization to achieve better model quality and training efficiency. Finally, the authors discuss how to customize the cost function and rearrange the model function to expand the model capabilities.

\section{Model formulation}

The goal of the grey-box thermal model is to use physicsbased parameters $\phi$ inside a model function $f$ to predict the future indoor air temperature vector $\widehat{T} . \widehat{T}$ consists of multiple forecasting horizons $h$ in the future. The model function uses current and historical observations, future weather forecasts and control variables as its inputs. The general form of this model is formulated as below:

$$
\widehat{T}=\left[\begin{array}{c}
T_{1} \\
T_{2} \\
\vdots \\
T_{h}
\end{array}\right]=\left[\begin{array}{c}
f\left(T_{0}, X_{0}, U_{0}\right) \\
f\left(T_{1}, X_{1}, U_{1}\right) \\
\vdots \\
f\left(T_{h-1}, X_{h-1}, U_{h-1}\right)
\end{array}\right]
$$

Where $X, U$ are exogenous state and control vectors that affect the thermal process of a building. The subscript 0 denotes variables from the current time, and $T_{1}, T_{2}, \ldots, T_{h}$ denote the forecasted indoor air temperature from the first forecast step to forecast step $h$. In essence this formulation requires one unified model function to iteratively predict future temperature. This approach may cause prediction errors to propagate, making forecasting results less reliable when the forecasting horizon becomes too large. In the scenario where a grey-box model is used, the model function $f$ should be consistent for all time steps since the physical processes being represented by the grey-box model remain unchanged.

As an alternative to mitigate the error propagation issue, some researchers (Srivastava, Pandey, \& Singh, 2016) have used dedicated models for each forecast step as below:

$$
\left[\begin{array}{c}
T_{1} \\
T_{2} \\
\vdots \\
T_{h}
\end{array}\right]=\left[\begin{array}{c}
f_{1}\left(T_{0}, X_{0}, U_{0}\right) \\
f_{2}\left(T_{0}, X_{0}, X_{1}, U_{0}, U_{1}\right) \\
\vdots \\
f_{h}\left(T_{0}, X_{0}, \ldots, X_{h-1}, U_{0}, \ldots, U_{h-1}\right)
\end{array}\right]
$$

As denoted in the above equation, for each forecast step a dedicated model function is responsible for using all previous inputs to predict the indoor air temperature. While this approach may provide improvements over the iterative process for longer horizon forecasts, it also increases the complexity of the overall forecasting model, making it harder to train. Some other researchers (Srivastava et al., 2016) have used a single black-box model for predicting all forecast steps in a multi-variate vector, and the author argues this approach requires a large covariance matrix instead of several smaller ones as formulated above. In this research the authors will compare the implementation of a grey-box thermal model using equation (1) against dedicated black-box models for different forecast steps based on equation (2).
The general form of the grey-box model function $f$ is:

$$
\begin{gathered}
T_{i}=f\left(T_{i-1}, T_{o a}, \psi\right)=T_{i-1}+\Delta t \sum_{n=1}^{\text {delay }} \phi_{o a, n}\left(T_{o a, i-n}-\right. \\
\left.T_{i-n}\right)+\Delta t \sum_{m=1}^{e x o} \sum_{n=1} \phi_{m, n} \psi_{m, i-n} \text { (3) }
\end{gathered}
$$

Where $T_{o a}$ is the outdoor air temperature, $\psi$ are the $m$ number of exogenous inputs represented in fractions, $\psi \in$ $[0,1]$ such as heating/cooling control and solar radiation. $\Delta t$ is the model time step, $\phi$ represents the reduced parameters for the grey-box model, and $n$ represent the number of trailing (historical) inputs for $T_{o a}$ and $\psi$ to reflect transient effects. The selection of $n$ for each of the inputs are discussed in the following feature selection section.

\section{Feature selection}

Similar to the model formulation step, there are multiple approaches to select the best inputs for $f$. This step is called feature selection, part of feature engineering, and is often practiced in machine learning problems. One common approach is to use information criteria, such as the Akaike information criterion (AIC) and the Bayesian information criterion (BIC), to determine relative quality of models constructed from different combinations of inputs (Bozdogan, 1987). This approach is sometimes called a wrapper method since it wraps around all possible subsets of input features. This method is usually very computationally intensive, since it requires all potential models to be trained with all possible subsets of features to evaluate them. The wrapper method is not selected in this research due to its low practicality and scalability.

Instead, the authors decided to use the so-called filter method to use proxy measures to prescreen the best input features and their delays for the thermal model. Since the thermal model itself is analogous to a linear additive model, Spearman correlation coefficients between $\Delta T$ and $T-T_{o a}, \psi$ are selected as the proxy. For nonlinear models, nonlinear measures such as mutual information (Fraser \& Swinney, 1986) can be used as the proxy instead.

Another approach, regularization, performs feature selection by including additional terms in the cost function to penalize additional features (Friedman, 2004). Still, this approach requires the initial training of the model to include all potential inputs, and is more suitable to black-box models, in which the filter method is less applicable due to model nonlinearity.

\section{Vectorization}

The iterative computation scheme used in equation (1) is rather inefficient on a modern computer, i.e. each forecast step needs to be computed iteratively for each prediction. To combat this, the authors propose two methods to improve the computation performance, as shown in equation (4) and (5). The first is to substitute the model function so that the entire $\hat{T}$ can be computed simultaneously. Still, substitution is usually not optimized in numerical computation. The best way to improve computation efficiency is to express everything in matrices. To this end, the model function can be vectorised into matrix form. Vectorization can be achieved by multiplying an augmented vector of $X$ and $U$ 
with the matrix $A . A$ is the Jacobian Matrix of the substituted model function, as in $A=\frac{\delta \hat{T}}{\delta x}=g(\phi)$. To further speed up the computation, the authors also used graphical processing units (GPU) to accelerate the matrix manipulation process.

substitution:

$$
\hat{T}=\left[\begin{array}{c}
f\left(T_{0}, X_{0}, U_{0}\right) \\
f\left(f\left(T_{0}, X_{0}, U_{0}\right), X_{1}, U_{1}\right) \\
\vdots \\
f\left(f\left(T_{h-1}, X_{h-1}, U_{h-1}\right), X_{h-1}, U_{h-1}\right)
\end{array}\right]
$$

vectorization:

$$
\widehat{T}=x A, \quad \text { where } x=\left[\begin{array}{l}
T_{0} \\
X \\
U
\end{array}\right], A=\frac{\delta \hat{T}}{\delta x}
$$

\section{Model training and cost function}

When training a model the cost function needs to be established to set the target for the model to be optimized on. Since the grey-box model is used to forecast future temperature, the model residuals of all forecast steps may need to be taken into consideration when calculating the cost function. Furthermore, since the model will be trained recursively, observations closer to the training time may be more important due to the underlying seasonal effects. To resolve these two potential requirements, the authors propose two weighting vectors to calculate the model cost $C(f)$ :

$$
C(f)=[\lambda(\widehat{T}-T)]^{2} \omega
$$

Here a quadratic loss function $(\widehat{T}-T)^{2}$ is used to calculate the residual matrix, $\lambda$ is the horizon weighting vector dictating how important each forecast step is to the total model accuracy, and $\omega$ is the observation weighting vector determining which observations are more dominant to fitting the data. Since quadratic loss is applied, the optimization problem is strictly convex.

To further illustrate the formulation of the cost function, an example is provided in Figure 1. $r_{a, b}$ is the residual for forecast step $b$ at time $a$. In this case, $r_{1,1}=\left(\widehat{T}_{1,1}-\right.$ $\left.T_{1}\right)^{2}, r_{1,2}=\left(\hat{T}_{1,2}-T_{2}\right)^{2}$, and so forth. On the left of the figure linear horizon weighting and linear observation weighting is used. On the right of the figure exponential

\begin{tabular}{|c|c|c|c|c|c|}
\hline$\omega$ & 1 & 1 & $\cdots$ & 1 & 1 \\
\hline 1 & $r_{1,1}$ & $r_{2,1}$ & $\cdots$ & $r_{9,1}$ & $r_{10,1}$ \\
\cline { 2 - 6 } 1 & $r_{1,2}$ & $r_{2,2}$ & $\cdots$ & $r_{9,2}$ & $r_{10,2}$ \\
\cline { 2 - 6 } 1 & $r_{1,3}$ & $r_{2,3}$ & $\cdots$ & $r_{9,3}$ & $r_{10,3}$ \\
\cline { 2 - 6 }$\lambda$ & \multicolumn{5}{|c}{} \\
\end{tabular}

observation weighting is used, where data closer to the time of training is more important. In addition, only the first two forecast steps are used for optimization while the third forecast step is discarded. While only using two forecasting steps may reduce the model accuracy, it could reduce the training time by not calculating the rest of the forecast steps at all during the model training process, in this case theoretically reducing the amount of training calculations by about one-third.

\section{Model rearrangement}

An additional reason to use a grey-box model is the easiness of rearranging the model function to approximate the required heating/cooling control or any other control term $\psi_{c}$ over the time step $\Delta t$, if the future indoor air temperature $T_{i}$ is predetermined:

$$
\psi_{c, i-1}=\frac{T_{i}-f\left(T_{i-1}, T_{o a}, \psi_{k}\right)}{\Delta t \phi_{c, i-1}}, \psi_{c, i-1} \notin \psi_{k}
$$

While such functionality is still possible with a nonlinear or a machine learning thermal model through backpropagation, it is usually difficult to program or isolate the effect of a single input term. In some black-box models where the model function is not reversible, separate models that solely predict control inputs have to be trained in order to achieve this task, which further increases the computation requirement.

\section{Case Study}

The data set used in this study consists of data from 564 dwellings located in Southern Ontario, Canada (Newsham, Pardasani, Grinberg, \& Bar, 2016). The dataset contained overall electricity consumption and thermostat data for 527 houses, motion/door (occupancy) data for 416 houses, and household characteristic data for 254 houses from March 2015 to February 2016. More than $80 \%$ of the dwellings are detached houses, the remainder being semi-detached houses or condos. All of the dwellings are primarily heated by natural gas and cooled by electricity through a central forced air system with floating on/off control. Data for each dwelling is collected from a smart (interval) electricity meter, a smart thermostat, multiple infrared occupancy sensors and door contact sensors. Variables recorded in this data set include: indoor air temperature, heating/cooling set point, heating/cooling runtime and system mode (heat/cool/off), electricity consumption, and a total number of occupancy

\begin{tabular}{|c|c|c|c|c|c|}
\hline$\omega$ & 1 & 2 & $\cdots$ & 8 & 9 \\
\hline 1 & $r_{1,1}$ & $r_{2,1}$ & $\cdots$ & $r_{9,1}$ & $r_{10,1}$ \\
\cline { 2 - 6 } 1 & $r_{1,2}$ & $r_{2,2}$ & $\cdots$ & $r_{9,2}$ & $r_{10,2}$ \\
\cline { 2 - 6 } & $r_{1,3}$ & $r_{2,3}$ & $\cdots$ & $r_{9,3}$ & $r_{10,3}$ \\
\cline { 2 - 6 }$\lambda$ & \multicolumn{5}{|c}{} \\
\hline
\end{tabular}

Figure 1: Example of empirical risk function for a training size of 10 observations and 3 time step forecast horizon. The left hand side uses linear weighting, and right hand size uses custom weighting. 
sensor firings. The temporal resolution of the data set is hourly. Since measurements from the sensors are collected and uploaded through each household's own wireless network, the temporal continuity is not guaranteed and missing data was not uncommon. On average $27 \%$ of the data is missing. The authors interpolated missing data less than 4 hours long, and then discarded the rest. For the weather data, hourly temperature, wind speed and relative humidity data are obtained through Environment Canada services (Environment Canada, n.d.), and hourly solar radiation is calculated using daily solar radiation data from NASA (Rosenzweig et al., 2014). The formulation of the greybox model for this study is presented as below:

$$
\begin{gathered}
T_{i}=T_{i-1}+\Delta t\left[\sum \phi_{o a, n}\left(T_{o a, i-n}-T_{i-n}\right)+\right. \\
\sum \phi_{o c c, n} \psi_{o c c, i-n}+\sum \phi_{\operatorname{rad}_{s}, n} \psi_{\text {rad }_{s}, i-n}+ \\
\sum \phi_{\text {rad }_{w}, n} \psi_{\text {rad }_{w}, i-n}+\sum \phi_{\text {rad }_{e}, n} \psi_{\text {rad }_{e}, i-n}+ \\
\left.\phi_{\text {heat }} \psi_{\text {heat }, i-1}+\phi_{\text {cool }} \psi_{\text {cool }, i-1}\right]
\end{gathered}
$$

The delay term $n$ for each of the exogenous inputs are determined by the spearman correlation with a threshold of 0.3 . For this problem $\Delta t$ is set to 3600 secs to reflect the one-hour data resolution. The model itself is implemented in Python, using scipy, numpy, (Jones, Oliphant, \& Peterson) sympy (Meurer et al., 2017) for formulation, and compiled to TensorFlow (Abadi et al., 2015) for GPU acceleration. To avoid local minima, a global optimization algorithm called differential evolution from scipy is used (Storn \& Price, 1997).

\section{Results}

Three major components of the results are presented: first the authors compare the computation efficiency of vectorization with iteration and substitution. Next, different choices of weighting functions $\lambda$ and $\omega$ and how they affect the accuracy of the grey-box models are evaluated. Then the authors compare the proposed greybox model against the other model strategies discussed in the methodology section. Finally, the authors examine the reduced physical parameters and demonstrate how to rearrange the thermal model to predict cooling demand.

\section{Vectorization}

Table 1 compares the processing time of different computation options. Iteration, substitution and vectorization are performed on an Intel i-5 $7600 \mathrm{~K}$ processor, and GPU acceleration is performed on an Nvidia GeForce RTX 2070. A single computation is the average time needed to predict a two-week temperature change with a four-hour horizon. The model training time is the average time required to recursively train the model with four-week historical data. Since the cost function is convex, gradient-based optimization is used.

As theorized in the methodology section, instead of iteration, adopting substitution to calculate all predictions simultaneously reduced computation time by more than one order of magnitude. Vectorising the model function further decreased the computation time by another order of magnitude. The GPU accelerated vectorization reduced computation time, but only by a smaller margin.
The reduction in computation time for model training is less significant than single computations, but still effective enough to justify the usage of vectorization. The difference between CPU-based vectorization and GPU acceleration is minimal since the size of the matrices are relatively small and GPU tends to scale well with very larger matrices. Yet for a multi-zone grey-box thermal model such as office buildings, GPU acceleration is worth investigating and may scale better.

Table 1: Computation time comparison

\begin{tabular}{|c|c|c|}
\hline $\begin{array}{c}\text { Computation } \\
\text { Method }\end{array}$ & $\begin{array}{c}\text { Average } \\
\text { Computation } \\
\text { Time }\end{array}$ & $\begin{array}{c}\text { Average } \\
\text { Model } \\
\text { Training } \\
\text { Time }\end{array}$ \\
\hline$\underline{\text { Iteration }}$ & $1,089 \mathrm{~ms}$ & Too long \\
\hline$\underline{\text { Substitution }}$ & $36 \mathrm{~ms}$ & $630 \mathrm{~ms}$ \\
\hline$\frac{\text { Vectorization }}{\underline{\text { (CPU) }}}$ & $1 \mathrm{~ms}$ & $63 \mathrm{~ms}$ \\
\hline$\frac{\text { Vectorization }}{\underline{\text { GPU })}}$ & $0.5 \mathrm{~ms}$ & $50 \mathrm{~ms}$ \\
\hline
\end{tabular}

\section{Model comparison}

Table 2 shows the root mean squared error (RMSE) of temperature predictions using different training weights $\lambda$ for the grey-box thermal model. Using the first two forecast horizons improved the overall forecasting accuracy, while the further inclusion of more forecast steps brings minimal improvements. Still, this indicates the requirement for using multiple forecasting steps, but not all horizons for model training. The following analysis uses the model trained with the first two forecast horizons. On average, a four-hour horizon has an error of $0.44^{\circ} \mathrm{C}$. The mean error is exaggerated by a few dwellings with very high unpredictability in temperature changes, and general uncertainties during the shoulder seasons. When dwellings with high uncertainties are excluded from the analysis, the mean RMSE is further reduced by $40 \%$ to $0.26^{\circ} \mathrm{C}$. The authors recommend future users of this approach to validate the models recursively before applying them, and to use alternative modelling techniques for dwellings with low grey-box model accuracy.

Table 2: RMSE of temperature prediction with different training weights

\begin{tabular}{|c|c|c|c|c|}
\hline $\begin{array}{c}\text { Training } \\
\text { weights }\end{array}$ & $\begin{array}{c}\text { 1-hr } \\
\text { horizon }\end{array}$ & $\begin{array}{c}\text { 2-hr } \\
\text { horizon }\end{array}$ & $\begin{array}{c}\text { 3-hr } \\
\text { horizon }\end{array}$ & $\begin{array}{c}\text { 4-hr } \\
\text { horizon }\end{array}$ \\
\hline$(1,0,0,0)$ & 0.19 & 0.31 & 0.42 & 0.49 \\
\hline $\mathbf{( 1 , 1 , 0 , 0 )}$ & $\mathbf{0 . 1 6}$ & $\mathbf{0 . 2 8}$ & $\mathbf{0 . 3 7}$ & $\mathbf{0 . 4 4}$ \\
\hline$(1,1,1,0)$ & 0.15 & 0.28 & 0.37 & 0.44 \\
\hline$(1,1,1,1)$ & 0.15 & 0.28 & 0.37 & 0.44 \\
\hline
\end{tabular}

Table 3 compares the accuracy of the proposed grey-box model with its black-box alternatives, specifically support vector regression (SVR). A naïve model, which uses the current observation as the future prediction, served as a 
benchmark. Single SVR uses equation (1) to calculate the future temperature iteratively, while dedicated SVR uses equation (2) to compute all forecast horizons simultaneously. All three methods achieved much better accuracy than the naïve model. Encouragingly, the proposed grey-box model achieved a similar level of performance as the dedicated SVR. Interestingly, single SVR based on equation (1) performed much worse than the other two models, which means that careful preparation and formulation are needed even for blackbox models.

Table 3: Comparison of grey-box model and black-box models, in terms of prediction RMSE

\begin{tabular}{|c|c|c|c|c|}
\hline $\begin{array}{c}\text { Forecast } \\
\text { RMSE } \\
\left({ }^{0} \mathrm{C}\right)\end{array}$ & $\begin{array}{c}\text { 1-hr } \\
\text { horizon }\end{array}$ & $\begin{array}{c}2 \text {-hr } \\
\text { horizon }\end{array}$ & $\begin{array}{c}\text { 3-hr } \\
\text { horizon }\end{array}$ & $\begin{array}{c}\text { 4-hr } \\
\text { horizon }\end{array}$ \\
\hline$\underline{\text { Naïve }}$ & 0.43 & 1 & 1 & 1.02 \\
\hline$\frac{\text { Proposed }}{\text { grey-box }}$ & 0.16 & 0.28 & 0.37 & 0.44 \\
\hline$\frac{\text { Single }}{\underline{\text { SVR }}}$ & 0.18 & 0.47 & 0.57 & 0.66 \\
\hline$\frac{\text { Dedicated }}{\underline{\text { SVR }}}$ & 0.18 & 0.28 & 0.36 & 0.42 \\
\hline
\end{tabular}

\section{Parameter estimates}

As covered earlier, one advantage of the grey-box model is the easiness to convert its model parameters into meaningful physical insights about the houses. Figure 2 provides an example of such an application. Maximum heat gain rate (HG Max) and maximum heat loss rate (HL Max) are estimated by applying the model parameters to the 5\% weather condition from the TMY2 file, while cooling capacity (CC) and heating capacity (HC) are parameters directly inside the reduced thermal model.

Upon examining the figures the author discovered a trend of undersized cooling capacity and over-sized heating capacity. This means that to combat a lack of cooling capacity, households either have to use larger sized AC units or use longer duty cycles through the cooling season, which is consistent with the predictions from the local jurisdiction (Ontario Energy Board, 2016). Furthermore, some dwellings are observed to have high heat loss potential but smaller heating capacity, which may lead to thermal comfort issues.

The grey-box model presents many other opportunities for parameter estimation and housing stock analysis, such as estimating a dwelling's orientation, occupant schedule, solar gains, its window-to-wall ratio, and other characteristics. However, this analysis requires a more complete dataset with ground-truth surveys to validate its effectiveness.

\section{Cooling load estimation}

The authors also tried to rearrange the model function to predict the cooling/heating needed to maintain the indoor air temperature. Figure 3 provides an example of such application for one dwelling during the summer. Overall the predicted and the actual cooling load agrees reasonably well, with an error of 0.21 . The authors are currently working on expanding this functionality with electricity models to perform automated load shifting for the residential dwellings. Future work is needed to further validate this application.

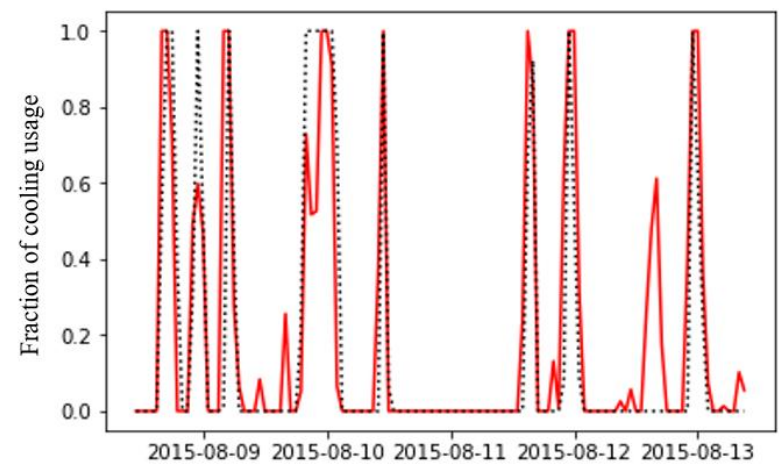

Figure 3: Example of cooling fraction prediction (red: predicted; black: actual
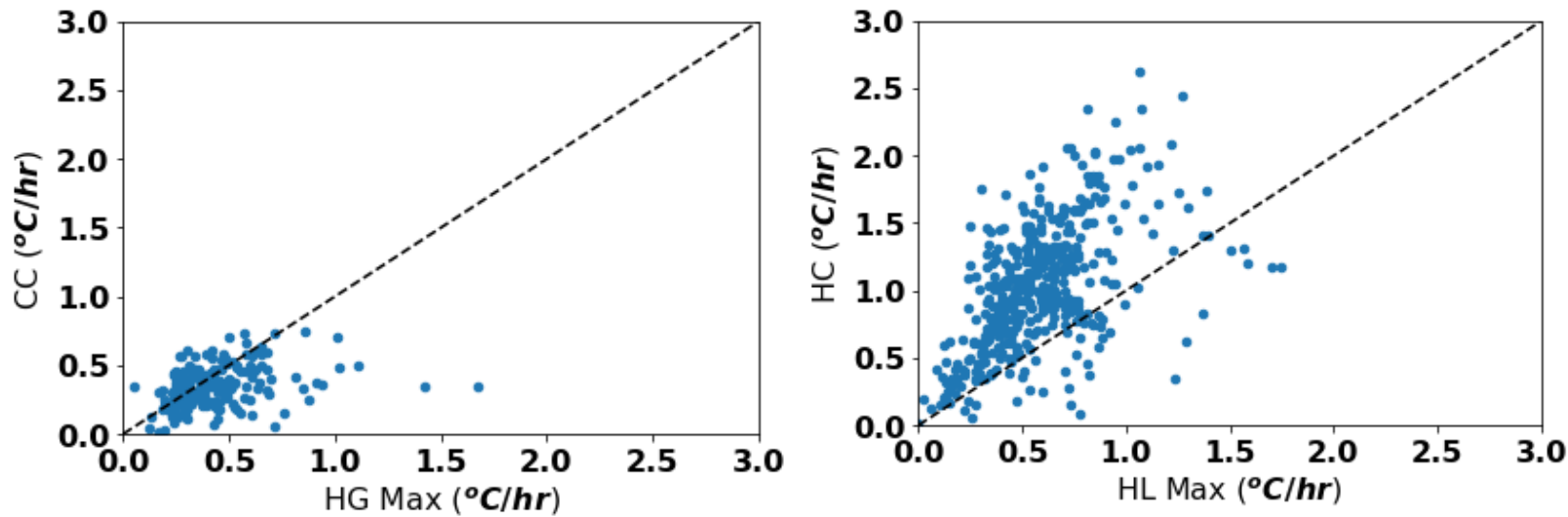

Figure 2: Estimated thermal characteristics of the dwellings, dotted line denotes perfect equipment sizing 


\section{Discussion and Future Work}

Overall the accuracy of the indoor air temperature forecast from the grey-box model performs at a similar level as the dedicated black-box model while being more interpretable. The proposed model also achieved more than $50 \%$ improvement over the naïve model. Results from cost function selection suggests that including two forecast steps in the training function provides a noticeable improvement over model accuracy, while including further forecast steps increases model training complexity and brings only diminishing returns. As a result, on average, the proposed grey-box model could forecast indoor air temperature with a 4-hour horizon with a $0.44^{\circ} \mathrm{C}$ uncertainty for the 500 dwellings included in the case study. A better $0.26^{\circ} \mathrm{C}$ uncertainty can be achieved by discarding dwellings with poor model fit. The authors have also tried to use future known set point as a predictor for indoor air temperature, but the prediction accuracy was poor. This further suggests that predicting the thermal response of a house given the vast contributors to uncertainty is a challenging task.

On implementing the grey-box model itself, the authors also demonstrated the importance of adopting vectorization when computing and optimizing the reduced thermal model. However, as the model computation time becomes faster, the time needed to preprocess the dataset starts to bottleneck the entire workflow.

The parameter estimation results suggest some inconsistencies with respect to equipment sizing and the actual cooling/heating demand. Based on this particular dataset in southern Ontario, Canada, under-sizing is generally observed for cooling equipment and over-sizing is more likely for heating equipment. This may provide some guidance in terms of future policies, such as to provide incentives to avoid equipment over-sizing, and to estimate the increase in future electricity demand due to households increasing cooling capacities to deal with the climate change patterns.

The issue of under-sizing and over-sizing also identified a potential application for model predictive control in this location. Model predictive control could mitigate the effect of under-sized cooling by pre-cooling the building to reduce the risk of uncomfortable indoor conditions, thus reducing the requirement for larger air conditioning units. On the other hand, it could also put a capacity usage threshold on over-sized equipment to reduce frequent on/off cycling and reduce peak demand, which is especially critical for electrically-heated dwellings in other locations.

Some proposed future work includes testing the proposed grey-box thermal modelling scheme with a longer forecast horizon, and determining the maximum possible forecast horizon until the model accuracy converges with the naïve model. With the integration with energy models it is also possible to produce model predictive control systems for individual households. Finally since the solar data used in this study is interpolated from coarse daily observations, it is worth investigating if an increase in data resolution would improve the overall reliability of the model.

\section{Conclusion}

This paper demonstrated various techniques for formulating and constructing a grey-box thermal model for residential buildings. The selection of the cost function heavily affects the accuracy of the thermal model, especially for longer prediction horizons. A properly constructed grey-box thermal model could perform as well as a black-box model for indoor temperature forecasting, with the added benefit of providing meaningful parameter estimates and control estimation. The authors also discussed the importance of vectorization to significantly improve model efficiency and reduce computation time, which is critical to practical applications. Some inherent limitations to thermal model observability still exist, such as uncertain heat transfer from occupant's actions and appliance cycling.

\section{Acknowledgement}

The authors would like to acknowledge High Performance Building Program at National Research Council for supporting this research. The authors would also like to thank Dr. Araz Ashouri, Dr. Adam Wills and Dr. Trevor Nightingale for providing feedback to this work. Finally, the lead author would like to thank his new born son Joey for giving him sleepless nights to think creatively.

\section{References}

Abadi, M., Agarwal, A., Barham, P., Brevdo, E., Chen, Z., Citro, C., ... Zheng, X. (2015). TensorFlow: Large-Scale Machine Learning on Heterogeneous Distributed Systems. In Google Research. https://doi.org/10.1080/09548980500300507

Bozdogan, H. (1987). Model selection and Akaike's Information Criterion (AIC): The general theory and its analytical extensions. Psychometrika, 52(3), 345-370. https://doi.org/10.1007/BF02294361

Braun, J., \& Chaturvedi, N. (2002). An Inverse Gray-Box Model for Transient Building Load Prediction. HVAC\&R Research, 8(1), 73-99. https://doi.org/10.1080/10789669.2002.10391290

Crawley, D., Sander, D. M., Cornick, S., \& Newsham, G. R. (1993). Development of a simple model to relate heating and cooling energy to building envelope thermal characteristics. Building Simulation 1993, 223-230. Retrieved from http://www.inive.org/Ibase_Search/search-detailairbase-001.asp?ID=101504

Déqué, F., Ollivier, F., \& Poblador, A. (2000). Grey boxes used to represent buildings with a minimum number of geometric and thermal parameters. Energy and Buildings, 31(1), 29-35. https://doi.org/10.1016/S0378-7788(98)00074-7

Eisenhower, B., O’Neill, Z., Narayanan, S., Fonoberov, V. A., \& Mezić, I. (2012). A methodology for metamodel based optimization in building energy models. Energy and Buildings, 47(April), 292-301. 
https://doi.org/10.1016/j.enbuild.2011.12.001

Environment Canada. (n.d.). Historical Data - Climate Environment and Climate Change Canada. Retrieved December 4, 2018, from http://climate.weather.gc.ca/historical_data/search _historic_data_e.html

Fraser, A. M., \& Swinney, H. L. (1986). Independent coordinates for strange attractors from mutual information. Physical Review A, 33(2), 1134-1140.

Friedman, J. H. (2004). Gradient Directed Regularization for Linear Regression and Classification. In Technical Report. Retrieved from stanford.

Gianniou, P., Reinhart, C., Hsu, D., Heller, A., \& Rode, C. (2018). Estimation of temperature setpoints and heat transfer coefficients among residential buildings in Denmark based on smart meter data. Building and Environment, 139(May), 125-133. https://doi.org/10.1016/j.buildenv.2018.05.016

Gunay, H. B., Bursill, J., Huchuk, B., O’Brien, W., \& Beausoleil-Morrison, I. (2014). Shortestprediction-horizon model-based predictive control for individual offices. Building and Environment, 82 , $408-419$ https://doi.org/10.1016/j.buildenv.2014.09.011

Jones, E., Oliphant, T., \& Peterson, P. (n.d.). \{SciPy\}: Open source scientific tools for \{Python\}.

Li, Q., Meng, Q., Cai, J., Yoshino, H., \& Mochida, A. (2009). Applying support vector machine to predict hourly cooling load in the building. Applied Energy, 86(10), $2249-2256$ https://doi.org/10.1016/j.apenergy.2008.11.035

Meurer, A., Smith, C. P., Paprocki, M., Čertík, O., Kirpichev, S. B., Rocklin, M., ... Scopatz, A. (2017). SymPy: symbolic computing in Python. PeerJ Computer Science, 3, e103. https://doi.org/10.7717/peerj-cs.103

Molina, D., Lu, C., Sherman, V., \& Harley, R. (2013). Model Predictive and Genetic Algorithm Based Optimization of Residential Temperature Control in the Presence of Time-Varying Electricity Prices. IEEE Transactions on Industry Applications, 49(3), 1137-1145.
Mustafaraj, G., Lowry, G., \& Chen, J. (2011). Prediction of room temperature and relative humidity by autoregressive linear and nonlinear neural network models for an open office. Energy and Buildings, 43(6), $1452-1460$ https://doi.org/10.1016/j.enbuild.2011.02.007

Newsham, G. R., Grinberg, Y., \& Bar, K. (2016). Remote energy auditing: Energy efficiency through smart thermostat data and control. ECEEE Summer Study Proceedings, 1003-1012.

Ontario Energy Board. (2016). Defining Ontario's Typical Electricity Customer. Toronto, Ontario.

Rosenzweig, C., Horton, R. M., Bader, D. A., Brown, M. E., DeYoung, R., Dominguez, O., ... Toufectis, K. (2014). Enhancing Climate Resilience at NASA Centers: A Collaboration between Science and Stewardship. Bulletin of the American Meteorological Society, 95(9), 1351-1363. https://doi.org/10.1175/BAMS-D-12-00169.1

Shi, Z., \& O’Brien, W. (2019). Sequential state prediction and parameter estimation with constrained dual extended Kalman filter for building zone thermal responses. Energy and Buildings, 183, 538-546. https://doi.org/10.1016/j.enbuild.2018.11.024

Srivastava, A. K., Pandey, A. S., \& Singh, D. (2016). Short-term load forecasting methods: A review. 2016 International Conference on Emerging Trends in Electrical Electronics \& Sustainable Energy Systems (ICETEESES), 130-138. https://doi.org/10.1109/ICETEESES.2016.758137 3

Storn, R., \& Price, K. (1997). Differential Evolution - A Simple and Efficient Heuristic for Global Optimization over Continuous Spaces. Journal of Global Optimization, 11(4), 341-359. https://doi.org/10.1175/JPO-D-17-0045.1

Wang, S., \& Xu, X. (2006). Simplified building model for transient thermal performance estimation using GA-based parameter identification. International Journal of Thermal Sciences, 45(4), 419-432. https://doi.org/10.1016/j.ijthermalsci.2005.06.009 